\title{
AGES OF GIANT ELLIPTICAL GALAXIES AS EVIDENCE FOR THE COSMOLOGICAL CONSTANT
}

\author{
YOUNG-WOOK LEE \\ Center for Space Astrophysics, Yonsei University \\ Shinchon 134, Seoul 120-749, Korea
}

\begin{abstract}
There is a compelling evidence for old ages of giant elliptical galaxies, about 3 Gyrs older than our Galaxy, from space ultraviolet observations and new population synthesis models. Alternative interpretation requires ad-hoc assumptions yet it produces poor fitting to observational data. If confirmed by future space UV mission, this would provide evidence for non-zero cosmological constant from time scale test.
\end{abstract}

\section{Introduction}

The surprising result of Hipparcos satellite suggests that the age of the oldest Galactic globular clusters is now reduced to 12 billion years, and the value of $H_{0}$ is reduced to $67-70 \mathrm{~km} / \mathrm{s} / \mathrm{Mpc}$ (Feast \& Catchpole 1997; Reid 1997; Gratton et al. 1997). If true, this would indicate that the infamous "age problem" is now solved, since this new globular cluster age and the value of $H_{0}$ are not in conflict with standard cosmological models without cosmological constant. But, before we rejoice too much, I would like to raise a possibility that globular clusters in our Galaxy may not be the oldest stellar population in the Universe, and even older population may exist in more massive galaxies, such as giant elliptical galaxies. This would be the case, if star formation (and metal enrichment) has proceeded much more rapidly and efficiently in giant elliptical galaxies (in denser environments). In this paper, we present a compelling evidence for such possibility from the analysis of space UV observations of elliptical galaxies with our new population synthesis models. 


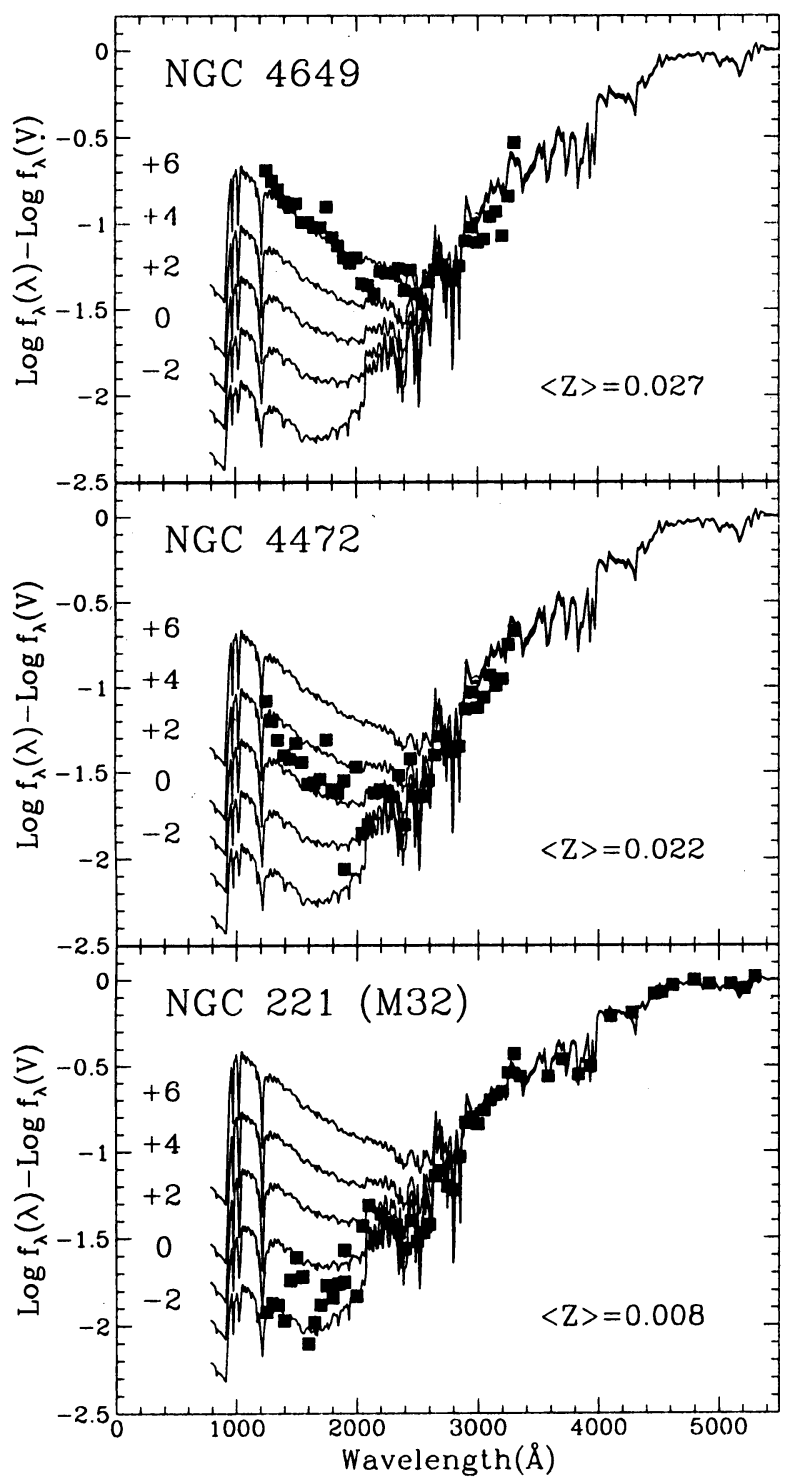

Fig. 1. Far-UV SEDs of three quiescent galaxies (Burstein et al. 1988) are compared with our composite models for $\Delta t=-2,0,+2,+4$, and $+6 \mathrm{Gyr}$ under different assumptions regarding the metallicity distribution function (and hence mean metallicity $<Z>$ ). Note that the age spread of several billion years among galaxies can reproduce the observed variation in UV upturn. 


\section{New Population Models \& Comparison with Observations}

Our population models supersede (and differ from) the previous models by including (1) the detailed modeling for the horizontal-branch (HB) and the post-HB stars, and (2) the effect of metallicity spread and corresponding variation in $\mathrm{H}-\mathrm{R}$ diagram morphology. Most of the previous works have been done without careful considerations of these two crucial effects. Other assumptions (Kurucz model atmosphere, Salpeter IMF, initial burst) and inputs are identical to previous models for elliptical galaxies.

An example of our population synthesis models is shown in Figure 1 of Park \& Lee (1997) for simple stellar populations like globular clusters. In the Park \& Lee (1997) models (see also Lee 1994), the most prominant change with age is the HB, in the sense that it gets bluer (or hotter) as age increases. This is nothing but the well known second parameter effect of the HB (see Lee et al. 1994) extrapolated to very old ages. As a result, the far-UV flux is dominated by HB and post-HB stars in our old metal-poor models. In the case of relatively metal-rich model, the morphology in the H-R diagram is shifted to red, mostly due to the effect of opacity. Thus, when age is increased, the HB gets bluer again, but it is not as hot as in the metal-poor models. Consequently, far-UV flux is not strong in metal-rich models, and only post-AGB stars contribute some UV flux (see Park \& Lee 1997). There is still some debate on the origin of second parameter effect of the HB, but now it is generally accepted that age is the most important second parameter that controls the morphology (mean temperature) of $\mathrm{HB}$ stars (see Lee et al. 1994).

If star formation in normal elliptical galaxies can be approximated as initial burst, a model elliptical galaxy is nothing but a composite of simpel population models, weighted according to the assumed metallicity distribution function. Figure 1 shows such composite models under different assumptions regarding the metallicity distribution function (and thus mean metallicity), where we can see that the UV flux (upturn) increases with increasing age, because the HB and post-HB stars gets hotter (bluer) with age. In the optical region, however, the spectral energy distribution (SED) is not very sensitive to age. In Figure 1, we have also compared the IUE spectra of elliptical galaxies with the model sequence that reproduce the observed mean metallicity of each galaxy. It is clear that our models reproduce the observed far-UV SED including $2500 \AA$ dip; and the systematic variation of UV upturn is understood in terms of age variation among galaxies. This and similar comparison in (far UV - V) color index vs. $M g_{2}$ diagram (see Fig. 5 of Park \& Lee 1997) suggest that old stars in giant elliptical galaxies are some 3-4 billion years older than those in spiral bulges of the Local Group, such as M31 and the Milky Way Galaxy. 
The alternative interpretation of the UV upturn phenomen was devised even before our models, which argue that the systematic variation of UV flux among galaxies is due to metallicity effect rather than age effect (Greggio \& Renzini 1990; Horch et al. 1992; Bressan et al. 1994). However, their models need some ad-hoc assumptions, such as enhanced stellar mass-loss in super metal-rich population, in order to make super metal-rich HB stars hot enough to produce UV flux. Also, recent observations from the Hopkins Ultraviolet Telescope aboard the Astro Space Shuttle mission (Ferguson et al. 1991; Brown et al. 1997) suggest that absorption features are relatively weak $(<[\mathrm{Fe} / \mathrm{H}]\rangle=-1)$. While this result is consistent with our models, where the UV flux comes both from metal-poor HB and postHB stars and metal-rich PAGB stars with the mean metallicity compatible to the observed absorption features, it is apparently in conflict with the alternative interpretation, unless the observed atmospheric abundances do not reflect the interior abundances due to diffusion processes (see Park \& Lee 1997). Furthermore, the metal-rich solution can not reproduce the observed UV SED including $2500 \AA$ dip (see Fig. 17 of Bressan et al. 1994), while our models reproduce the observations, both SEDs and absorption features (see Park \& Lee 1997). Future observations from next generation space UV instruments will undoubtedely help to clarify this problem, but we feel that above arguments already provide some discriminant against alternative interpretation.

\section{Implications}

To summarize, our work has two profound implications:

(1) Our result implies a prolonged epoch of galaxy formation, in the sense that the initial epoch of star formation in more massive galaxies occured earlier than that of smaller galaxies (perhaps, as a result of more efficient star formation and metal-enrichment in denser environments).

(2) The globular clusters in our Galaxy may not be the oldest population in the Universe. According to our result, the oldest stellar population in the Universe must be found at the center of giant elliptical galaxies. They are estimated to be some 3-4 billion years older than the oldest globular clusters in the Milky Way Galaxy. Even if the reader is prepared to accept recent Hipparcos result on globular cluster ages $(\sim 12 G y r)$, this suggests that the age of the Universe is older than 15 billion years, which can be considered as evidence for non-zero cosmological constant.

\section{References}

Bressan, A., Chiosi, C., \& Fagotto, F. 1994, ApJS, 94, 63

Brown, T. M, Ferguson, H. C., Davidsen, A. F., \& Dorman, D. 1997, ApJ, 482, 685 
Burstein, D., Bertola, F., Buson, L. M., Faber, S. M., \& Lauer, T. R. 1988, ApJ, 328, 440

Feast, M. W. \& Catchpole, R. M. 1997, MNRAS, 286, L1

Ferguson, H. C. et al. 1991, ApJ, 382, L69

Gratton, R. G. et al. 1997, ApJ, 491,749

Greggio, L, \& Renzini, A. 1990, ApJ, 364, 35

Horch, E., Demarque, P., \& Pinsonneault, M. 1992, ApJ, 388, L53

Lee, Y.-W. 1994, ApJ, 430, L113

Lee, Y.-W., Demarque, P., \& Zinn, R. 1994, ApJ, 423, 248

Park, J.-H. \& Lee, Y.-W. 1997, ApJ, 476, 28

Reid, I. N. 1997, AJ, 114, 161 